\title{
Ischemic perconditioning on mesenteric ischemia/reperfusion injury in rats
}

\author{
Lainy Carollyne da Costa Cavalcante ${ }^{1} \mathbb{D}$, Giannini Medeiros Rodrigues ${ }^{1} \mathbb{D}$, Rubens Fernando Gonçalves \\ Ribeiro Júnior ${ }^{2^{*}}$ (D), Andrew Moraes Monteiro ${ }^{3}$ (D), Ananda Vitória Barros Suzuki Damasceno ${ }^{3}$ (D), \\ Rodrigo Paracampo Couteiro ${ }^{4}$ (D), Edson Yuzur Yasojima ${ }^{4}$ (D), Marcus Vinicius Henriques Brito ${ }^{5}$ (D), \\ Sandro Percário ${ }^{5}$ \\ 1. MD. Centro Universitário do Estado do Pará (CESUPA) - Belem (PA), Brazil. \\ 2. Fellow Master degree. Postgraduate Program in Surgery and Experimental Research - Universidade do Estado do Pará (UEPA) - Belem-PA, Brazil. \\ 3. MD. Universidade do Estado do Pará (UEPA) - Belem (PA), Brazil. \\ 4. PhD, Full Professor, Head. Division Surgical Abilities - Universidade do Estado do Pará (UEPA) - Belem (PA), Brazil. Conception, design, and scientific \\ content of study, critical revision. \\ 5. PhD, Full Professor. Universidade Federal do Pará (UFPA) - Belem (PA), Brazil.
}

\begin{abstract}
Purpose: To evaluate if the perconditioning affects the antioxidant capacity in mesenteric ischemia and reperfusion injury. Methods: Twenty-one Wistar rats were assigned into three groups, as follows: Sham, IR and rPER. The animals were subjected to mesenteric ischemia for $30 \mathrm{~min}$. rPER consisted of three cycles of 5-min hindlimb ischemia followed by 5 min hindlimb perfusion at the same time to mesenteric ischemic period. After 5 minutes, blood and $5 \mathrm{~cm}$ of terminal ileum were harvested for thiobarbituric acid reactive substances (TBARS) and Trolox equivalent antioxidant capacity (TEAC) measurement. Results: rPER technique was able to reduce intestinal tissue TBARS levels $(p<0.0001)$, but no statistic difference was observed in blood levels between groups, although it was verified similar results in rPER and Sham group. rPER technique also enhanced TEAC levels in both blood $(p=0.0314)$ and intestinal tissue $(p=0.0139)$, compared to IR group. Conclusions: rPER appears as the most promising technique to avoid IR injury. This technique reduced TBARS levels in blood and intestinal tissue and promoted the maintenance of antioxidant defense in mesenteric acute injury.
\end{abstract}

Key words: Mesenteric Ischemia. Reperfusion. Mesenteric Vascular Occlusion. Oxidative Stress. Rats.

*Corresponding author: rubensfernandojr@gmail.com | (55 91)98133-7502

Received: May 06, 2021 | Review: July 03, 2021 | Accepted: Aug 09, 2021

Conflict of interest: Nothing to declare.

Research performed at Laboratory of Experimental Surgery (LCE), Universidade do Estado do Pará (UEPA), Belem-PA, Brazil. 


\section{Introduction}

The blood flow interruption of organs and tissues and their subsequent restoration causes a cascade of molecular events known as ischemia and reperfusion syndrome (IR), perceived in polytrauma and organ transplants ${ }^{1}$, triggering the formation of reactive oxygen species (ROS) immune activation and endothelial dysfunction ${ }^{2}$.

Even though most of the research focus on myocardial infarction, the importance of IR syndrome covers any organ that has a compromised blood supply and subsequently restored ${ }^{3}$, especially the intestine, an organ that is very sensitive to $\mathbf{I R}^{4}$.

The intestinal ischemia process presents a diagnostic challenge, because its clinical presentation often occurs in a non-specific way, in addition to the difficulty in recognizing the conditions in which the patient is, before intestinal necrosis occurs. The incidence of intestinal ischemia is increasing, and the mortality rates observed in hospital environments have remained high over the past few decades, ranging from 60 to $80 \%$.

This fact is related to the priority of perfusion to other organs in response to circulatory shock. Even after volume restoration, vasoconstriction persists at all levels of intestinal microvasculature, due to the effects of multiple agents, such as vasoactive substances, and factors derived from the endothelium ${ }^{6}$.

Therefore, several experimental treatment methods have been studied and applied in animal models in order to mitigate the tissue damage caused by intestinal IR. Among these methods, ischemic perconditioning seems to be the most promising strategy for reducing reperfusion injury after ischemia, increasing the tolerance of the intestine against the damage caused by ischemia and reperfusion syndrome ${ }^{7,8}$.

Schmidt et al. ${ }^{9}$ reported the method of remote ischemic conditioning (rPER), which consists on the application of remote ischemic conditioning, by means of a tourniquet performed on the hind limb of pigs, during the main ischemic time, which was effective in preventing reperfusion injury in myocardial ischemia. This protective effect was corroborated by further studies involving myocardial ischemia, and the technique has been expanded to cerebral ischemia ${ }^{10-12}$.

Thus, the aim of this study was to evaluate if the perconditioning affects the antioxidant capacity in mesenteric ischemia and reperfusion injury.

\section{Methods}

All experiments were performed in accordance with the Brazilian law for scientific use of animals (Law No. 11.794/08) and the National Institutes of Health (NIH) guide for the care and use of laboratory animals (NIH Publications No. 8,023, revised 1978). The research was approved by Animal Use and Care Committee of Universidade do Estado do Pará (UEPA) (Protocol 01/2016).
Twenty-one (12-15 wk) male Wistar rats, weighing 290 $\pm 19 \mathrm{~g}$, were used in this study. The animals were kept in a vivarium of the Experimental Surgery Laboratory, UEPA, with a controlled temperature, light, humidity, and noise. Water and food were provided ad libitum.

\section{Experimental protocol}

The animals were randomly assigned into the following three groups ( $n=7$ for each group):

- Sham group (Sham): the same surgical procedure as in the remaining groups was performed, but no mesenteric ischemia was induced;

- Ischemia and reperfusion group (IR): mesenteric ischemia was induced for $30 \mathrm{~min}$, followed by reperfusion without any form of conditioning;

- Remote perconditioning group (rPER): mesenteric ischemia was simultaneously followed by remote ischemic perconditioning. rPER consisted of three cycles of 5-min left hindlimb ischemia followed by 5-min left hindlimb reperfusion (Fig. 1). Hindlimb ischemia was achieved using an elastic rubber band tied around the thigh of the left leg ${ }^{8,13}$. The remote ischemic conditioning technique was completed at the same time as the end of the main ischemia ${ }^{13-15}$, with subsequent reperfusion of 5 minutes.

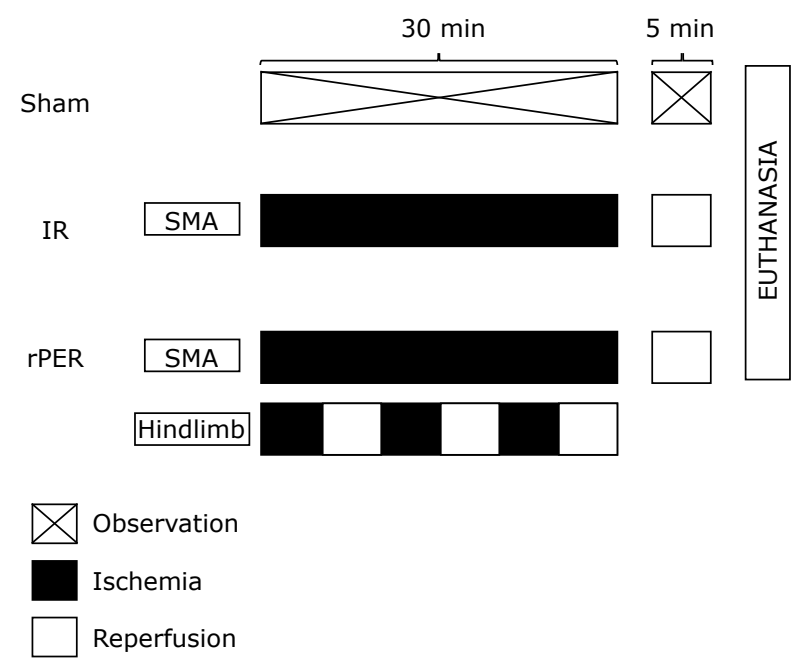

IR: ischemia and reperfusion; rPER: remote preconditioning; SMA: superior mesenteric artery.

Figure 1 - Experimental design. Sham group was not submitted to ischemic conditioning.

\section{Surgical procedures}

All surgical procedures were performed in anesthesia (ketamine hydrochloride and xylazine hydrochloride 60 and $6 \mathrm{mg} / \mathrm{kg}$, respectively, i.p.). Through a median longitudinal laparotomy, superior mesenteric artery was 
occluded by microsurgical clamp application, leading to mesenteric ischemia.

After the ischemia and conditioning protocols, animals were submitted to painless death induced after 5 min of mesenteric reperfusion. Then, blood sample was obtained via puncture of superior mesenteric vein, and $5 \mathrm{~cm}$ of terminal ileum was harvested for biochemical analysis. Subsequently, the animals were euthanized by lethal anesthetic doses.

\section{Laboratory parameters}

The samples were homogenized and then immediately centrifuged at 3,000 rpm for $10 \mathrm{~min}$. After centrifugation, samples were directly transferred to Eppendorf tubes and stored at $-80^{\circ} \mathrm{C}$ until assayed. Thiobarbituric acid (TBARS) and the Trolox equivalent antioxidant capacity (TEAC) levels were determined. Biochemical parameters were assessed at the Laboratory for Research on Oxidative Stress, at Universidade Federal do Pará.

\section{Statistics}

The software BioEstat ${ }^{\odot} 5.0$ was used. All data were expressed as means \pm standard deviation. Analysis of variance, followed by Tukey post hoc test correction, was performed. Statistical significance was assumed at $p<0.05$.

\section{Results}

No animal died during the anesthesia, procedures, or reperfusion period. The ischemic perconditioning technique reduced levels of TBARS in blood sample. IR group $(0.018 \pm 0.003)$ had the highest TBARS serum levels in relation to other groups, while Sham group $(0.015 \pm 0.002)$ and $r P E R$ group $(0.017 \pm 0.003)$ presented similar results and had no difference among them $(p=0.1917)$, even presenting a lower value in relation to IR group (Fig. 2).

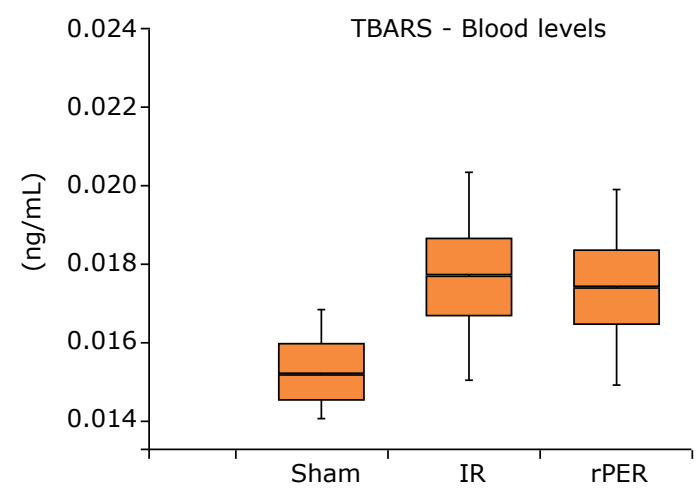

TBARS: Thiobarbituric acid; IR: ischemia and reperfusion; rPER: remote preconditioning.

Figure 2 - TBARS blood levels $(\mathrm{ng} / \mathrm{mL}$ ) according to groups. Analysis of variance (Tukey post hoc test).
rPER caused an increase in TEAC serum values (Fig. 3). IR group $(0.616 \pm 0.033)$ had the lowest TEAC values compared to other groups, while rPER $(0.929 \pm 0.014)$ obtained values close to Sham (0.942 \pm 0.043$)$. There was a statistically significant difference between the Sham $(p<0.0001)$ and the $r P E R(p<0.0001)$ groups in relation to the IR one.

$$
\text { TEAC - Blood levels }
$$

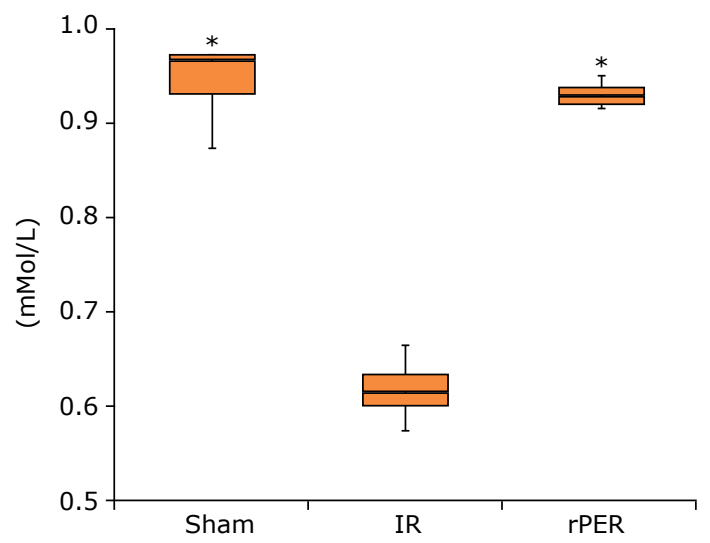

TEAC: Trolox equivalent antioxidant capacity; IR: ischemia and reperfusion; rPER: remote preconditioning; ${ }^{*} p<0.05$ vs. IR. Analysis of variance (Tukey post hoc test).

Figure 3 - TEAC blood levels (mMol/L) according to groups.

In intestinal tissue, there was a statistically significant difference in TBARS levels (Fig. 4) between Sham ( $p=0.0226$ ) and rPER ( $p=0.0139)$ groups compared to IR group. TEAC blood levels (Fig. 5) showed the same effect between Sham $(p=0.0228)$ and $\mathrm{rPER}(\mathrm{p}=0.0314)$ groups in relation to IR group.

TBARS - Intestinal tissue

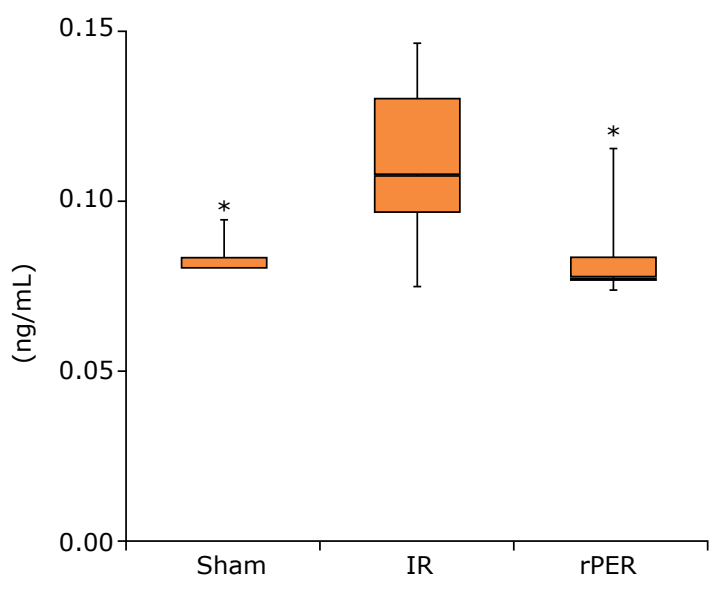

TBARS: Thiobarbituric acid; IR: ischemia and reperfusion; rPER: remote preconditioning; ${ }^{*} p<0.05$ vs. IR. Analysis of variance (Tukey post hoc test).

Figure 4 - TBARS intestinal tissue levels $(\mathrm{ng} / \mathrm{mL}$ ) according to groups. 


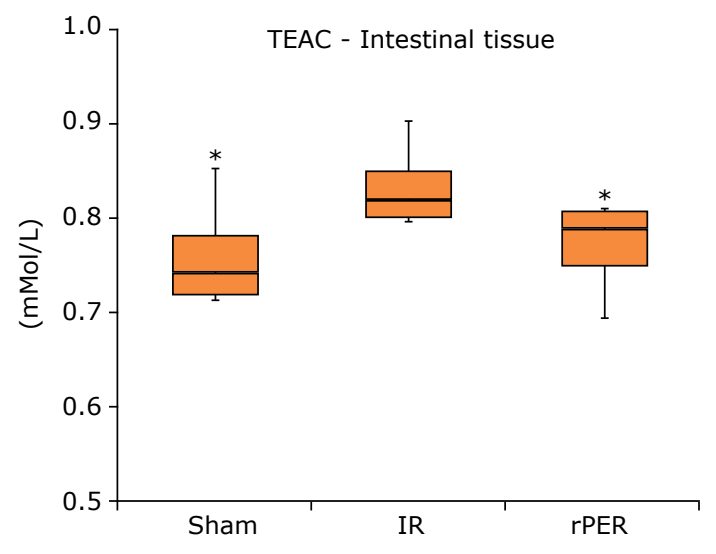

TEAC: Trolox equivalent antioxidant capacity; IR: ischemia and reperfusion; rPER: remote preconditioning; ${ }^{*} p<0.05 \mathrm{vs}$. IR. Analysis of variance (Tukey post hoc test).

Figure 5 - TEAC intestinal tissue levels (mMol/L) according to groups.

The data corresponding to the TBARS and TEAC values measured in blood and intestinal tissue are shown in Table 1.

Table 1 - Blood and intestine TBARS and TEAC values according to the groups.

\begin{tabular}{cccccc}
\multirow{2}{*}{ Group } & & \multicolumn{2}{c}{ TBARS $(\mathbf{n g} / \mathbf{m L})$} & \multicolumn{2}{c}{ TEAC $(\mathrm{mMol} / \mathrm{L})$} \\
\cline { 3 - 6 } & & Blood & Intestine & Blood & Intestine \\
\hline \multirow{2}{*}{ Sham } & 7 & $0.015 \pm$ & $0.091 \pm$ & $0.942 \pm$ & $0.526 \pm$ \\
& & 0.002 & $0.014^{*}$ & $0.043^{*}$ & $0.118^{*}$ \\
IR & 7 & $0.018 \pm$ & $0.112 \pm$ & $0.616 \pm$ & $0.668 \pm$ \\
& & 0.003 & 0.027 & $0.033^{*}$ & 0.085 \\
rPER & 7 & $0.017 \pm$ & $0.084 \pm$ & $0.929 \pm$ & $0.546 \pm$ \\
& & 0.003 & $0.014^{*}$ & $0.014^{*}$ & $0.091^{*}$ \\
\hline
\end{tabular}

TBARS: Thiobarbituric acid; TEAC: Trolox equivalent antioxidant capacity; IR: ischemia and reperfusion; rPER: remote preconditioning; ${ }^{*} p<0.05$, vs. IR group.

\section{Discussion}

The study of IR is very important, as its deleterious effects can aggravate the clinical conditions of patients undergoing complex surgeries (cardiac, vascular, transplantation, strangulated hernias, and neonatal necrotizing enterocolitis), as well as in certain emergency situations, such as trauma, extensive burns, hemorrhagic shock and septic shock ${ }^{14,16,17}$.

Knowing that even short periods of mesenteric ischemia can damage the intestinal mucosa, strategies have been developed to minimize its deleterious effects, such as preconditioning $^{7}$ and post-conditioning ${ }^{17,18}$.

However, no studies have been found yet using remote ischemic conditioning technique associated to mesenteric IR injury, in order to assess whether this technique is really capable of attenuating IR injuries in the intestine, while perconditioning was already effective in cases of myocardial ${ }^{14}$, cerebral $^{20}$ and renal ${ }^{13}$ ischemia injury.

The data presented showed that ischemic conditioning was able to reduce oxidative stress, approaching the values of the Sham group. Interestingly, there was no statistical difference between the IR and rPER groups, probably due to the short time of reperfusion, not being enough to alter oxidative stress at the systemic level, or the process of free radical formation being extremely acute. Despite that, it can be said that after performing the ischemic conditioning technique, based on previous studies, there was an additional protection promoted by the execution of remote ischemic conditioning $8,10,14$.

Regarding the TBARS dosage, there were no statistically significant differences between groups in the blood samples. This fact suggests that there was no systemic repercussion of local changes in this model, or that the great variability of behaviors presented individually by the animals studied may have masked this repercussion ${ }^{21,22}$.

On the other hand, in the samples of intestinal tissue, there was a difference between sham and perconditioning groups when compared to the IR, suggesting that the ischemic-reperfusion process to which the animals in the IR group were submitted resulted in an increase in the levels of free radicals, as well as, consequently, in the involvement of oxidative stress ${ }^{23}$.

The possible activity of antioxidant enzymes increases such as catalase, superoxide dismutase and glutathioneperoxidase in these groups may be associated with reduction in the degree of lesion of mesenteric IR. It may also have been responsible for the protective activity of the conditioning, which promotes a decrease of neutrophil bearing and activation of nitric oxide to the site of inflammation ${ }^{8,13}$.

To measure the formation of ROS, TEAC was evaluated in the animals' blood, which showed how strongly the production of these free radicals can consume the endogenous antioxidant reserve. It was noticed that the ischemic conditioning technique was able to avoid the consumption of these antioxidant substances, allowing to affirm that the technique provides greater protection preventing the formation of ROS, since their values were close to those of the sham group ${ }^{24}$, demonstrating the consumption of systemic antioxidant defenses to mitigate the oxidative damage imposed by the ischemia and reperfusion syndrome to animals.

Furthermore, the significant increase in antioxidant capacity observed at tissue level in the IR group can be explained by the fact that it was subjected to the most intense ischemic injury when compared to rPER group, 
validating that the conditioning technique proved to be effective in the process of decreased tissue damage.

Although many studies show that increasing antioxidant capacity occurs as a protective factor, it can be said that an increase in this parameter, as observed in the IR group, when evaluated in a short reperfusion period, works as an indicator of greater tissue damage acute by the intrinsic mechanisms of ischemia and reperfusion, such as the formation of EROS ${ }^{21,25}$.

In addition, a similar behavior was observed when comparing the TEAC values between the sham and rPER groups, which allows to suppose that this technique was able to maintain the antioxidant capacity at normal levels, since the sham group was not submitted to any intervention ${ }^{26}$.

Oxidative stress involves the reactive oxygen speciesmediated oxidative degradation of the components of cellular membrane phospholipids followed by formation of peroxy radicals and finally lipid peroxides, that are metabolized, via $\beta$-oxidation pathway, to malondialdehyde $(\mathrm{MDA})^{27}$. In the stomach, for instance, remote ischemic conditioning (RIC) stimulus to the heart or liver significantly reduced gastric mucosal injury, improved gastric blood flow, and suppressed plasma proinflammatory cytokine levels in rat model of gastric ischemic injury ${ }^{28}$, having never previously performed a study in the intestine.

These findings confirm the effective participation of oxidative stress in this model of mesenteric ischemia and reperfusion syndrome in rats and that the application of the isolated ischemic perconditioning technique can protect animals from associated oxidative changes.

\section{Conclusion}

The perconditioning technique was effective in reducing the damage caused by ROS from the mesenteric ischemia and reperfusion syndrome, observed by the decrease in blood and tissue levels of TBARS and in the maintenance of antioxidant capacity, assessed by TEAC, at levels close to parameters of normality.

\section{Author's contribution}

Conception, design, and scientific content of the study: Couteiro RP, Yasojima EY, Brito MVH and Percário $S$; Interpretation of data: Cavalcante LCC, Rodrigues GM, Monteiro AM and Damasceno AVBS; Technical procedures: Cavalcante LCC, Rodrigues GM, Monteiro AM and Damasceno AVBS; Statistical analysis: Ribeiro Júnior RFG; English language: Ribeiro Júnior RFG; Manuscript preparation: Cavalcante LCC, Rodrigues GM, Ribeiro Júnior
RFG, Monteiro AM and Damasceno AVBS; Critical revision: Couteiro RP, Yasojima EY, Brito MVH and Percário S.

\section{Data availability statement}

Data will be available upon request.

\section{Funding}

Not applicable.

\section{Acknowledgments}

To Deivid Ramos dos Santos for providing language help and writing assistanc, and for the assistance in statistical analysis provided by Mariseth Carvalho de Andrade, Master of Surgery and Experimental Research Program at UEPA.

\section{References}

1. Chen G, Thakkar M, Robinson C, Doré S. Limb remote ischemic conditioning: mechanisms, anesthetics, and the potential for expanding therapeutic options. Front Neurol 2018;9:1-18. https://doi.org/10.3389/fneur.2018.00040

2. Glorieux C, Zamocky M, Sandoval J, Verrax J, Calderon PB. Regulation of catalase expression in healthy and cancerous cells. Free Radic Biol Med. 2015;87:84-97. https://doi. org/10.1016/j.freeradbiomed.2015.06.017

3. Mccafferty K, Forbes $\mathrm{S}$, Thiemermann $\mathrm{C}$, Yaqoob $\mathrm{M}$. The challenge of translating ischemic conditioning from animal models to humans: the role of comorbidities. Dis Model Mech. 2014;7:1321-33. https://doi.org/10.1242/ dmm.016741

4. Yamamoto S, Tanabe M, Wakabayashi G, Shimazu M, Matsumoto K, Kitajima $M$. The role of tumor necrosis factor-alpha and interleukin-1 beta in ischemia-reperfusion injury of the rat small intestine. J Surg Res. 2001;99;13441. https://doi.org/10.1006/jsre.2001.6106

5. Clair DG, Beach JM. Mesenteric ischemia. N Engl J Med. 2016;374:959-68. https://doi.org/10.1056/ NEJMra1503884

6. Granger DN, Kvietys PR. Reperfusion injury and reactive oxygen species: The evolution of a concept. Redox Bio. 2015;6:524-51. https://doi.org/10.1016/j. redox.2015.08.020

7. Mallick IH, Yang W, Winslet, MC, Seifalian AM. Ischemiareperfusion injury of the intestine and protective strategies against injury. Dig Dis Sci. 2004;49:1359-77. https://doi. org/10.1023/B:DDAS.0000042232.98927.91

8. Costa FLS, Teixeira RKC, Yamaki VN, Valente AL, Silva AMF, Brito MVH, Percário S. Remote ischemic conditioning temporarily improves antioxidant defense. J Surg Res. 2016;200:105-9. https://doi.org/10.1016/j. jss.2015.07.031 
9. Schmidt $M R$, Smerup $M$, Konstantinov IE, Shimizu $M$, Li J, Cheung M, White PA, Kristiansen SB, Sorensen K, Dzavik V, Redington N, Kharbanda RK. Intermittent peripheral tissue ischemia during coronary ischemia reduces myocardial infarction through a KATP-dependent mechanism: first demonstration of remote ischemic preconditioning. Am J Physiol Heart Circ Physiol. 2007;292;1883-90. https://doi. org/10.1152/ajpheart.00617.2006

10. Zhu SB, Liu Y, Zhu Y, Yin GL, Wang RP, Zhang Y, Zhu J, Jiang $W$. Remote preconditioning, perconditioning, and postconditioning: a comparative study of their cardioprotective properties in rat models. Clinics. 2013;68:263-8. https://doi.org/10.6061/CLINICS/2013(02)OA22

11. Lou Z, Wang AP, Duan XM, Hu GH, Song GL, Zuo ML, Yang ZB. Upregulation of NOX2 and NOX4 mediated by TGF- $\beta$ signaling pathway exacerbates cerebral ischemia/ reperfusion oxidative stress injury. Cell Physiol Biochem. 2018;46:2103-13. https://doi.org/10.1159/000489450

12. Parks DA, Granger DN. Contributions of ischemia and reperfusion to mucosal lesion formation. Am J Physiol. 1986;250(6 Pt 1):G749-53. https://doi.org/10.1152/ ajpgi.1986.250.6.G749

13. Costa FLS, Yamaki VN, Teixeira RK, Feijó DH, Valente AL, Carvalho LTF, Yasojima EY, Brito MVH. Perconditioning combined with postconditioning on kidney ischemia and reperfusion. Acta Cir Bras. 2017;32:599-606. https://doi. org/10.1590/s0102-865020170080000001

14. Baranyai T, Nagy CT, Koncsos G, Onódi Z, Károlyi-Szabó M, Makkos A, Varga ZV, Ferdinandy P, Giricz Z. Acute hyperglycemia abolishes cardioprotection by remote ischemic perconditioning. Cardiovasc Diabetol 2015;14:15161. https://doi.org/10.1186/s12933-015-0313-1

15. Oliveira RC, Brito MVH, Ribeiro Júnior RFG, Oliveira LOD, Monteiro AM, Brandão FMV, Cavalcante LCC, Gouveia, $\mathrm{EHH}$, Henriques HYB. Influence of remote ischemic conditioning and tramadol hydrochloride on oxidative stress in kidney ischemia/reperfusion injury in rats. Acta Cir Bras. 2017;32:229-35. https://doi.org/10.1590/s0102865020170030000007

16. Yamaguchi T, Izumi $Y$, Nakamura $Y$, Yamazaki T, Shiota M, Sano S, Tanaka M, Osada-Oka M, Shimada K, Miura $K$, Yoshiyama $M$, Iwao $H$. Repeated remote ischemic conditioning attenuates left ventricular remodeling via exosome-mediated intercellular communication on chronic heart failure after myocardial infarction. Int J Cardiol 2015;178:239-46. https://doi.org/10.1016/j. ijcard.2014.10.144

17. Ji YY, Wang ZD, Wang SF, Wang BT, Yang ZA, Zhou XR, Lei NN, Yue WN. Ischemic preconditioning ameliorates intestinal injury induced by ischemia-reperfusion in rats. World J Gastroenterol. 2015;21;8081-8. https://doi.org/10.3748/ wjg.v21.i26.8081

18. Jang HS, Kim JI, Kim J, Park JW, Park KM. Angiotensin II removes kidney resistance conferred by ischemic preconditioning. Biomed Res Int. 2014;2014:602149. https://doi.org/10.1155/2014/602149
19. Gholampour F, Roozbeh J, Janfeshan S, Karimib Z. Remote ischemic per-conditioning protects against renal ischemiareperfusion injury via suppressing gene expression of TLR4 and TNF- $\alpha$ in rat model. Can J Physiol Pharmacol. 2019;97:112-9. https://doi.org/10.1139/cjpp-2018-0543

20. Donato $M$, Buchholz $B$, Rodríguez $M$, Pérez $V$, Inserte J, García-Dorado D, Gelpi RJ. Role of the parasympathetic nervous system in cardioprotection by remote hindlimb ischaemic preconditioning. Exp Physiol 2013;98:425. https://doi.org/10.1113/expphysiol.2012.066217

21. Watanabe $Y$, Murdoch C, Sano S, Ido Y, Bachschmid MM, Cohen RA, Matsui R. Glutathione adducts induced by ischemia and deletion of glutaredoxin-1 stabilize HIF$1 \alpha$ and improve limb revascularization. Proc Natl Acad Sci U S A. 2016;113:6011-6. https://doi.org/10.1073/ pnas.1524198113

22. Percário S. Prevention of oxidative stress in renal ischemiareperfusion syndrome in rats with nutritional antioxidant supplementation. Rev Nutr. 2010;23:259-67. https://doi. org/10.1590/S1415-52732010000200009

23. Brito MVH, Yasojima EY, Percário $S$, Ribeiro Junior RFG, Cavalcante LCC, Monteiro AM, Couteiro RP, Rodrigues IAS, Santos HAG. Effects of hypertonic saline solution associated to remote ischemic perconditioning in kidney ischemia/ reperfusion injury in rats. Acta Cir Bra. 2017;32:211-8. https://doi.org/10.1590/s0102-865020170030000005

24. Costa FL, Yamaki VN, Gonçalves TB, Coelho JV, Percário S, Brito MVH. Combined remote ischemic perconditioning and local postconditioning on liver ischemia-reperfusion injury. J Surg Res. 2014;192:98-102. https://doi. org/10.1016/j.jss.2014.05.046

25. Ren C, Li S, Wang B, Han R, Li N, Gao J, Li X, Jin K, Ji X. Limb remote ischemic conditioning increases Notch signaling activity and promotes arteriogenesis in the ischemic rat brain. Behav Brain Res. 2018;340:87-93. https://doi. org/10.1016/j.bbr.2016.10.036

26. Huang Y, Shan J, Wang C, Ma J, Li D, Li L, Li S, Li Y. Can ischemic preconditioning alone really protect organs from ischemia reperfusion injury in transplantation. Transplant Immunol. 2009;20:127-31. https://doi.org/10.1016/j. trim.2008.08.002

27. Gholampour F, Khangah L, Vatanparast J, Karbalaei-Heidari HR, Owji SM, Bahaoddini A. The role of nitric oxide in the protective action of remote ischemic per-conditioning against ischemia/reperfusion-induced acute renal failure in rat. Iran J Basic Med Sci. 2018;21(6):600-6. https://doi. org/10.22038/IJBMS.2018.25810.6354

28. Koike Y, Li B, Ganji N, Zhu H, Miyake H, Chen Y, Lee C, Lok MJ, Zozaya C, Lau E, Lee D, Chusilp S, Zhang Z, Yamoto M, Wu RY, Inoue $M$, Uchida K, Kusunoki $M$, Delgado-Olguin $P$, Mertens L, Daneman A, Eaton S, Sherman PM, Pierro A. Remote ischemic conditioning counteracts the intestinal damage of necrotizing enterocolitis by improving intestinal microcirculation. Nat Commun. 2020;11:4950. https://doi. org/10.1038/s41467-020-18750-9 